\title{
Estado nutricional e consumo alimentar de idosos participantes dos grupos Hiperdia
}

\author{
Nutritional status and food consumption of elderly participants in the Hiperdia groups
}

Estado nutricional y consumo de alimentos de los participantes mayores em los grupos de Hiperdia

Jéssica de Oliveira Campos ${ }^{1 *}$, Adriana Maria da Silva², Cathleen Sandy de Amorim Rocha², Camilla Peixoto Santos Rodrigues ${ }^{2}$, Marcela de Albuquerque Melo ${ }^{1}$, Eduila Maria Couto Santos ${ }^{2}$, Antonio Flaudiano Bem leite ${ }^{2}$, Michelle Figueiredo Carvalho².

\section{RESUMO}

Objetivo: Avaliar o impacto das atividades educativas do programa Hiperdia sobre o estado nutricional (EN) e o consumo alimentar de idosos participantes. Métodos: Estudo transversal quantitativo, realizado com 50 idosos dos grupos Hiperdia de um município da zona da mata de Pernambuco. Os idosos foram subdivididos em dois grupos: Grupo A, com atividades quinzenais realizadas para um público fixo, e grupo B, com atividades mensais e um público rotativo. Foram avaliados o índice de massa corporal e as circunferências da cintura, do braço e da panturrilha. O consumo alimentar foi avaliado por questionário de frequência alimentar. Resultados: Não foram encontradas diferenças no EN entre os grupos avaliados. Contudo, em relação ao consumo alimentar, observou-se um maior consumo de verduras, legumes e frango no grupo $A$. $O$ grupo $B$ apresentou um maior consumo de carnes vermelhas e manteiga/margarina, o que sugere um maior consumo de gorduras nesse grupo. Conclusão: Conclui-se que o grupo $A$, apresentou maior ingestão de verduras, legumes e frango e menor consumo de gorduras. Assim, sugere-se que as atividades educativas quando realizadas de forma regular favorecem a melhora no consumo alimentar de idosos.

Palavras-chave: Avaliação nutricional, Doença crônica, Envelhecimento, Educação em saúde.

\begin{abstract}
Objective: To evaluate the impact of Hiperdia program educational activities on nutritional status (NS) and food consumption of elderly participants. Methods: Quantitative cross-sectional study, carried out with 50 elderly people from the Hiperdia groups in a municipality in the forest area of Pernambuco. The elderly were divided into two groups: Group A, with biweekly activities carried out for a fixed audience, and Group B, with monthly activities and a rotating audience. Body mass index and waist, arm and calf circumferences were evaluated. Food consumption was assessed using a food frequency questionnaire. Results: No differences were found in the NS between the groups evaluated. However, in relation to food consumption, there was a higher consumption of vegetables, legumes and chicken in group A. Group B showed a higher consumption of red meat and butter/margarine, which suggests a higher consumption of fats in this group. Conclusion: it was concluded that group A had a higher intake of vegetables, legumes and chicken and less consumption of fats. Thus, it is suggested that educational activities, when carried out on a regular basis, favor the improvement in food consumption of the elderly.
\end{abstract}

Key words: Nutritional assessment, Chronic disease, Aging, Health education.

\footnotetext{
${ }^{1}$ Universidade Federal de Pernambuco (UFPE), Recife - PE. *E-mail: Jessica.oliveiracampos@ufpe.br

${ }^{2}$ Universidade Federal de Pernambuco, Centro acadêmico de Vitória (UFPE-CAV), Vitória de Santo Antão - PE.
}

SUBMETIDO EM: 4/2021

ACEITO EM: 4/2021

PUBLICADO EM: 5/2021 


\section{RESUMEN}

Objetivo: Evaluar el impacto de las actividades educativas del Hiperdia sobre el estado nutricional (EN) y el consumo de alimentos de los ancianos participantes. Métodos: Estudio cuantitativo transversal, realizado con 50 ancianos de los grupos Hiperdia en un municipio de la zona forestal de Pernambuco. Se formaron dos grupos: Grupo A, con actividades quincenales y público fijo, y Grupo B, con actividades mensuales y público rotativo. Se evaluó el índice de masa corporal y las circunferencias de cintura, brazos y pantorrillas. El consumo de alimentos se evaluó mediante un cuestionario de frecuencia alimentaria. Resultados: No se encontraron diferencias en el EN entre los grupos. Sin embargo, en relación al consumo de alimentos, hubo mayor consumo de verduras, legumbres y pollo en el grupo A. El grupo B mostró mayor consumo de carnes rojas y mantequilla/margarina, lo que sugiere mayor consumo de grasas. Conclusión: El grupo A presentó mayor ingesta de verduras, legumbres y pollo y menor consumo de grasas. Así, se sugiere que las actividades educativas, cuando se realizan de forma regular, favorecen la mejora en el consumo de alimentos de los ancianos.

Palabras clave: Evaluación nutricional, Enfermedad crónica, Envejecimiento, Educación para la salud.

\section{INTRODUÇÃO}

O envelhecimento está associado a um processo biológico de declínio geral na capacidade intrínseca do indivíduo ao longo do tempo (OMS, 2015). As alterações endócrinas e metabólicas decorrentes do processo de envelhecimento incluem: aumento da resistência vascular, diminuição da frequência cardíaca, acloridria, redução da motilidade intestinal e de enzimas digestivas, fragilidade do sistema imunológico e perda de massa óssea e de massa muscular (FECHINE BRA e TROMPIERI NO, 2012). Essas alterações estão associadas à maior suscetibilidade a Doenças Crônicas Não Transmissíveis (DCNT) como: Hipertensão Arterial (HAS) e Diabetes Mellitus (DM) (SILVA TA, et al., 2020).

As DCNT constituem um problema de saúde pública, que exerce nos serviços de saúde um aumento na demanda por internações, medicações, exames e cuidados contínuos (SIMIELI I, et al., 2019). No Brasil a taxa de mortalidade por DCNT em 2018 representou quase $70 \%$ dos óbitos no país (BRASIL, 2018). Os principais fatores de risco para DCNT em idosos incluem: sedentarismo, tabagismo, consumo excessivo de álcool, hábitos alimentares inadequados e o excesso de peso (PODMELLE R e ZIMMERMANN RD, 2018).

Quando não controladas a HAS e o DM estão associadas a complicações de saúde entre os quais se destacam: infarto agudo do miocárdio, acidente vascular encefálico (AVE), amputações, cegueira e danos a função renal. Essas complicações acarretam um comprometimento da saúde do idoso e uma redução na qualidade de vida dos mesmos (CARVALHO AN, 2018).

As atividades desenvolvidas na atenção primária à saúde (APS) possuem como foco a promoção à saúde e a prevenção de doenças e agravos. Um estudo realizado por Silva GSM et al. (2021) observou que um programa de intervenção de educação em saúde na APS promoveu uma melhoria nos níveis de atividade física, na pressão arterial e nos indicadores antropométricos em idosos hipertensos. Além disso, a APS destaca-se como um ambiente propício para o desenvolvimento de grupos de educação em saúde, que promovam o autocuidado e o empoderamento do processo saúde e doença de idosos portadores de DCNT (DA SILVA IS, et al., 2020). Sendo assim, destaca-se o papel da APS e o seu impacto na prevenção de uma sobrecarga dos demais níveis de atenção à saúde (ROCHA TAH e SILVA NC, 2019).

Diante da complexidade e do impacto das DCNT nos serviços de saúde, o Ministério da Saúde elaborou o plano de reorganização da atenção à HAS e ao DM, conhecido como Hiperdia, o qual possui o objetivo de acompanhar o tratamento e a assistência aos portadores dessas patologias na APS (BRASIL, 2001). O Hiperdia é um programa que estimula nos usuários o autocuidado e a corresponsabilização pela saúde, através de: reuniões com ações educativas, estímulo à realização de atividades físicas, consultas médicas agendadas e entrega de medicamentos (SILVA JVF, et al., 2015). 
Nesse cenário, ressalta-se a importância do monitoramento dos fatores de risco e da prevalência das DCNT, bem como das ações e atividades desenvolvidas no território. Esse monitoramento possui o objetivo de direcionar as políticas públicas que atuam nas áreas de promoção à saúde e contribuem para um estilo de vida mais saudável (BRASIL, 2011). Sendo assim, o objetivo deste estudo foi avaliar o impacto das atividades educativas do grupo Hiperdia sobre o estado nutricional e o consumo alimentar de idosos participantes em um município da zona da mata de Pernambuco.

\section{MÉTODOS}

Estudo transversal quantitativo, realizado com 50 idosos ( $\geq 60$ anos) portadores de HAS e/ou DM que participam dos grupos Hiperdia de um município da zona da mata de Pernambuco. Foram excluídos os participantes que apresentaram patologias que afetam o estado nutricional e o consumo alimentar como: Síndrome da Imunodeficiência Adquirida (SIDA), Hepatopatias e Câncer. O estudo foi aprovado pelo comitê de ética em pesquisa com seres humanos do Centro de Ciências da Saúde - Universidade Federal de Pernambuco ( $\mathrm{N}^{\circ}$ 2.656.913). Todos os participantes assinaram o Termo de Consentimento Livre Esclarecido (TCLE) e os princípios contidos na declaração de Helsinque da associação médica mundial (revisada em 2000) foram respeitados.

Os participantes foram subdivididos em dois grupos, grupo $A$ e grupo $B$, devido às diferenças na execução das atividades educativas. $O$ grupo $A$ realiza atividades educativas quinzenais para participantes fixos vinculados ao Hiperdia, enquanto o grupo $B$ realiza atividades educativas mensais, na sala de espera da unidade para hipertensos e diabéticos, contudo o público é composto por diferentes faixas etárias e rotativo. Ressalta-se que ambos os grupos são acompanhados pela APS, na qual são realizadas consultas e distribuição dos medicamentos para o tratamento da HAS e do DM. A coleta de dados foi realizada por meio de visitas domiciliares, juntamente aos Agentes Comunitários de Saúde (ACS). Foram coletados dados do estado nutricional, do consumo alimentar e da situação sociodemográfica dos idosos participantes.

$\mathrm{Na}$ avaliação antropométrica foram coletados: peso, altura, índice de massa corporal (IMC), circunferência da panturrilha (CP) e da cintura (CC). Para a aferição do peso corporal utilizou-se uma balança eletrônica tech line com capacidade de $150 \mathrm{Kg}$. Os indivíduos se posicionaram no centro da balança, utilizando roupas leves e descalços (BRASIL, 2011). A altura foi estimada através da equação de Chumlea WC, et al. (1985). Para a aferição da altura do joelho utilizou-se uma fita inelástica da marca Sanny $(150 \mathrm{~cm})$. O indivíduo foi posicionado sentado, formando um ângulo de $90^{\circ}$ com o joelho, em seguida mediu-se a distância entre a borda superior da patela até o calcanhar (VITOLO MR, 2015). O IMC foi obtido pela razão entre o peso (Kg) e a altura (metros) elevada ao quadrado, utilizou-se a classificação proposta por Lipstchz D (1994) que considera a faixa de 22 a $27 \mathrm{Kg} / \mathrm{m}^{2}$ como eutrofia e as variações abaixo e acima dessa faixa correspondem ao baixo peso e ao excesso de peso (sobrepeso e obesidade), respectivamente.

As circunferências foram aferidas com o auxílio de uma fita métrica inelástica da marca Sanny $(150 \mathrm{~cm})$. A CC foi medida no ponto médio entre a última costela e a crista ilíaca, com o indivíduo em pé e com ausência de roupa na região. Foi considerado como risco cardiovascular elevado e muito elevado valores de $\geq 80$ e $\geq 88$ para mulheres e $\geq 94 \mathrm{e} \geq 102$ para homens, respectivamente (VITOLO MR, 2015). A CP foi aferida com o idoso sentado, com o joelho flexionado, formando um ângulo de $90^{\circ}$. Para a classificação considerou-se valores $\geq$ $31 \mathrm{~cm}$ adequados para ambos os sexos, os valores inferiores foram considerados indicativos de perda de massa muscular (VITOLO MR, 2015).

O consumo alimentar foi avaliado por meio de dois Questionários de Frequência Alimentar (QFA). O QFA - ELSA (versão reduzida), validado para a população adulta e idosa brasileira em um estudo realizado por Mannato LW (2013) contendo 76 alimentos agrupados em sete grupos alimentares; e o QFA utilizado por Piovesana PM (2015) para avaliar o consumo de alimentos fontes de sódio. Para a análise e interpretação do QFA foram elaborados escores que variaram de 9 a 0 indicando de forma decrescente a frequência do consumo de cada alimento, ou seja, nessa representação o 9 indica a maior frequência (2 ou mais vezes ao dia), enquanto o 0 indica a menor frequência de consumo (nunca ou quase nunca). Após a conversão da frequência alimentar em escores, realizou-se a identificação dos alimentos mais consumidos pelos 
participantes, através do somatório dos escores correspondentes a cada alimento e a cada grupo alimentar, com o auxílio da ferramenta do Excel. Em seguida foram selecionados os 3 alimentos mais consumidos e o somatório que corresponde ao consumo total dos alimentos de cada grupo alimentar.

O consumo em gramas ( $\mathrm{g}$ ) de sódio foi obtido através da fórmula indicada por Piovesana PM (2015), a porção consumida ( $\mathrm{g}$ ) é multiplicada pela frequência alimentar e posteriormente dividido pelos 30 dias do mês. No caso dos temperos prontos/caldo de carne, o valor obtido é dividido pelo número de pessoas que consomem a refeição para a obtenção da estimativa do consumo individual. O consumo de sódio obtido também foi representado em gramas de sal, considerando que $1 \mathrm{~g}$ de sal contém $400 \mathrm{mg}$ de sódio (SBC, 2020).

As análises estatísticas foram realizadas no software estatístico epilnfo versão 7.2.2.6. Realizou-se o teste de Kolmogorov-smirnnov para avaliar a normalidade dos dados. As variáveis que apresentaram distribuição normal foram apresentadas na forma de média e desvio padrão. As demais variáveis foram apresentadas por meio de porcentagens. Na comparação entre as médias, dos grupos A e B, foram realizados os testes de Mann-Whitney, Qui-Quadrado e Mantel Haenszel. Adotou-se o valor de significância < 0,05.

\section{RESULTADOS E DISCUSSÃO}

Os grupos foram compostos por idosos de ambos os sexos, em sua maior parte mulheres, solteiras/viúvas, com o ensino fundamental incompleto, aposentadas e com a renda familiar $\leq 1$ salário-mínimo. O grupo $\mathrm{A}$ apresentou melhor participação nas atividades educativas do Hiperdia, bem como uma menor média de idade quando comparado ao grupo B. Quanto as patologias todos os participantes apresentaram hipertensão e/ou diabetes, sendo a frequência de idosos hipertensos semelhantes entre os grupos, o grupo B apresentou maior número de idosos diabéticos, e o grupo $\mathrm{A}$ um maior número de idosos hipertensos e diabéticos (Tabela 1).

Tabela 1 - Características sociodemográficas dos grupos A e B de idosos participantes das atividades rupais do Hiperdia de um município da zona da mata de Pernambuco, 2018.

\begin{tabular}{|c|c|c|c|c|c|c|}
\hline \multirow{4}{*}{$\begin{array}{l}\text { Participação no } \\
\text { Hiperdia }\end{array}$} & \multirow[t]{2}{*}{ Variáveis Sociodemográfica } & \multicolumn{2}{|c|}{$\begin{array}{c}\text { Grupo A } \\
n=25\end{array}$} & \multicolumn{2}{|c|}{$\underset{\substack{\text { Grupo } \\
n=25}}{ }$} & \multirow[t]{2}{*}{ Valor $\mathbf{p}$} \\
\hline & & $\mathbf{N}$ & $\%$ & $\mathbf{N}$ & $\%$ & \\
\hline & Muito baixa/Baixa & 4 & 16 & 17 & 68 & \multirow{2}{*}{0,0002 * } \\
\hline & Muito boa/Boa & 21 & 84 & 8 & 32 & \\
\hline & Hipertensão & 14 & 56 & 14 & 56 & \multirow{3}{*}{0,53 ** } \\
\hline \multirow[t]{2}{*}{ Patologias } & Diabetes & 1 & 4 & 5 & 20 & \\
\hline & Hipertensão e diabetes & 10 & 40 & 6 & 24 & \\
\hline \multirow{2}{*}{ Sexo } & Feminino & 23 & 92 & 20 & 80 & \multirow{2}{*}{0,22 * } \\
\hline & Masculino & 2 & 8 & 5 & 20 & \\
\hline \multirow{2}{*}{ Escolaridade } & Analfabeto & 9 & 36 & 10 & 40 & \multirow{2}{*}{$0,77^{*}$} \\
\hline & Fundamental Incompleto & 16 & 64 & 15 & 60 & \\
\hline \multirow{2}{*}{ Renda Familiar } & s1 salário-Mínimo & 14 & 56 & 12 & 48 & \multirow{2}{*}{$0,57^{*}$} \\
\hline & $\geq 2$ Salários-Mínimos & 11 & 44 & 13 & 52 & \\
\hline \multirow{2}{*}{ Estado Civil } & Solteiro/ Viúvo & 14 & 56 & 15 & 60 & \multirow{2}{*}{$0,77^{*}$} \\
\hline & Casado & 11 & 44 & 10 & 40 & \\
\hline \multirow{2}{*}{ Ocupação } & Do lar & 8 & 32 & - & - & \multirow{2}{*}{0,002 * } \\
\hline & Aposentado & 17 & 68 & 25 & 100 & \\
\hline Moradores & Mora sozinho & 0 & 0 & 3 & 12 & $0,07^{*}$ \\
\hline \multicolumn{2}{|c|}{ Idade (Média \pm Desvio padrão) } & 68,4 & $\pm 6,73$ & 74,8 & $\pm 8,07$ & $0,007 \#$ \\
\hline
\end{tabular}

Legenda: * teste Mantel Haenszel; * ${ }^{* *}$ teste Qui- quadrado; \# Mann-Whitney/Wilcoxon two-Sample Test/.

Fonte: Campos JO, et al., 2021.

As características da amostra deste estudo são semelhantes ao estudo de Andrade RKO, et al. (2015), que também avaliou participantes de um grupo de promoção à saúde. A participação de idosos em atividades grupais de educação em saúde pode ser afetada por falta de interesse na temática abordada, dificuldade de 
acesso aos locais e o não conhecimento do grupo e dos profissionais envolvidos (ARAÚJO LSA, et al., 2017). Sendo assim, a menor participação dos idosos do grupo $B$ pode estar associada à ausência de uma rotina fixa para a realização das atividades e a idade, uma vez que idades avançadas dificultam a locomoção de participação nas atividades grupais (ARAÚJO LSA, et al., 2017).

Destaca-se que ambos os grupos são compostos predominantemente por mulheres, esse achado pode estar associado à construção histórica de que o homem é considerado forte e viril, que não necessita de cuidados e dificilmente adoece, o que contribui para a menor procura por serviços de saúde pelo sexo masculino (BOTTON A, et al., 2017).

Quanto ao estado nutricional não foram encontradas diferenças entre os grupos (Tabela 2). O IMC indica que mais de $50 \%$ dos idosos, em ambos, os grupos apresentam um quadro de excesso de peso. Esse dado reforça o estudo realizado por Cardozo NR, et al. (2017), no qual grande parte dos idosos avaliados apresentou excesso de peso (54,6\%). A utilização do IMC é amplamente difundida por ser um método de fácil aplicação e baixo custo, contudo a sua utilização em idosos tem sido questionada por não considerar as mudanças na estatura e na distribuição da gordura corporal provocadas pelo envelhecimento. Diante disso, utilizam-se pontos de corte diferentes para essa faixa etária com o objetivo de prevenir a desnutrição (SILVEIRA EA, et al., 2020).

Tabela 2 - Avaliação Nutricional entre os grupos A e B de idosos participantes das atividades grupais do Hiperdia de um município da zona da mata de Pernambuco, 2018.

\begin{tabular}{|c|c|c|c|c|c|c|}
\hline \multirow{4}{*}{ CP ${ }^{1}$} & \multirow{2}{*}{ Classificação } & \multicolumn{2}{|c|}{ Grupo A n=25 } & \multicolumn{2}{|c|}{ Grupo B n=25 } & \multirow{2}{*}{ Valor $p$} \\
\hline & & $\mathbf{N}$ & $\%$ & $\mathbf{N}$ & $\%$ & \\
\hline & Baixo & 2 & 8 & 2 & 8 & 100 * \\
\hline & Adequado & 23 & 92 & 23 & 92 & 1,00 \\
\hline \multirow{2}{*}{$\mathrm{CC}^{2}$} & Risco aumentado de DCV 4 & 2 & 8 & 4 & 16 & \\
\hline & Risco muito aumentado de DCV 4 & 23 & 92 & 21 & 84 & 0,38 \\
\hline \multirow{3}{*}{$\mathrm{IMC}^{3}$} & Baixo peso & 3 & 12 & 1 & 4 & \\
\hline & Eutrofia & 9 & 36 & 10 & 40 & $0,51^{* *}$ \\
\hline & Excesso de peso & 13 & 52 & 14 & 56 & \\
\hline
\end{tabular}

Legenda: *teste Mantel Haenszel; ** teste Qui- quadrado; ${ }^{1}$ Circunferência da Panturrilha; ${ }^{2}$ Circunferência da Cintura; ${ }^{3}$ Índice de Massa Corporal; ${ }^{4}$ Doença Cardiovascular.

Fonte: Campos JO, et al., 2021.

A CP indica normalidade em $92 \%$ dos idosos, sugerindo uma adequada manutenção da massa muscular nesses indivíduos. Contudo, Souza IP, et al. (2018) observou que idosos com excesso de peso podem apresentar CP normal, mas, não necessariamente apresentarão um bom desempenho muscular. Desta forma sugere-se que sejam realizados novos estudos para avaliar o desempenho muscular entre os grupos.

Quanto ao risco de Doenças Cardiovasculares (DCV), todos os participantes possuem algum risco indicado pela CC. Nos grupos A e B, respectivamente, $92 \%$ e $84 \%$ dos idosos possuem o risco considerado como muito elevado para DCV. Esse resultado corrobora com o estudo realizado Souza AM, et al. (2020) no qual $73,5 \%$ dos participantes apresentaram risco elevado para DCV. Os estudos têm demonstrado que mulheres idosas apresentam uma maior tendência ao aumento do risco cardiovascular indicado pela CC (OLIVEIRA VB, et al., 2019; SOUZA AM, et al., 2020). No estudo realizado por Souza AM, et al. (2020) com idosos, em um município da Bahia, observou que apesar do valor médio da CC ser maior em homens, as mulheres apresentaram maior risco para o desenvolvimento de DCV de acordo com a CC.

O envelhecimento está associado ao maior acúmulo de gordura corporal e redução na massa muscular (FALSARELLA GR, et al., 2014). O acúmulo de gordura corporal também sofre alterações em sua distribuição passando a ser, em indivíduos idosos, predominantemente na região abdominal, especialmente no sexo feminino (CARDOZO NR, et al., 2017). A obesidade abdominal em mulheres é correlacionada à deficiência hormonal ocasionada pela menopausa que favorece alterações no padrão de gordura corporal que deixa de ser gluteofemoral e passa a ser abdominal (OLIVEIRA HHB, et al., 2020). Esse fator pode ter influenciado os 
resultados obtidos neste estudo que indicam maior risco cardiovascular, segundo a CC, no grupo $A$, uma vez que nesse grupo o percentual de mulheres.

Os grupos A e B não apresentaram diferenças em relação ao consumo alimentar de sódio. Os alimentos considerados fontes de sódio que apresentaram o maior consumo em ambos os grupos foram: salsicha, temperos prontos e caldos de carne (Tabela 3).

Tabela 3 - Consumo de alimentos fontes de sódio, consumo de sódio e de sal (gramas) entre os grupos A e B de idosos participantes das atividades grupais do Hiperdia de um município da zona da mata de Pernambuco, 2018.

\begin{tabular}{cccccc}
\hline \multirow{2}{*}{ Alimento fonte } & \multicolumn{2}{c}{$\begin{array}{c}\text { Grupo A } \\
\mathbf{n = 2 5}\end{array}$} & \multicolumn{2}{c}{$\begin{array}{c}\text { Grupo B } \\
\mathbf{n = 2 5}\end{array}$} & \multirow{2}{*}{ Valor p } \\
\cline { 2 - 5 } & Média (Score) & DP & Média (Score) & DP & \\
\hline Salsicha & 1,08 & $\pm 1,65$ & 1,12 & $\pm 1,81$ & 0,78 \\
Tempero pronto & 1,60 & $\pm 2,29$ & 1,76 & $\pm 2,66$ & 1,00 \\
Caldo de carne & 1,84 & $\pm 2,42$ & 2,48 & $\pm 2,63$ & 0,39 \\
Consumo de sódio $(\mathrm{g})$ & 0,329 & $\pm 0,328$ & 0,295 & $\pm 0,29$ & 0,97 \\
Consumo de sal $(\mathrm{g})$ & 0,82 & - & 0,737 & - & - \\
\hline
\end{tabular}

Legenda: *Mann-Whitney/Wilcoxon Two-Sample Test.

Fonte: Campos JO, et al., 2021.

A Sociedade Brasileira de Cardiologia (SBC, 2020), estabelece as recomendações de consumo de sódio para a população, considerando essa recomendação os valores obtidos neste estudo representam cerca de $16,5 \%$ e 14,7\% da recomendação diária de sódio nos grupos A e B, respectivamente. Piovessana PM, (2015) ressalta que o consumo de sódio quantificado através do QFA- Sódio não foi capaz de identificar a ingestão real desse micronutriente, visto que o questionário possui uma lista de alimentos fixos e não contabiliza o consumo do sal de adição. Sendo assim, faz-se necessário que outros métodos sejam utilizados concomitantemente ao QFA-sódio como, por exemplo, o recordatório de 24 horas. Enfatizamos que o QFAsódio não inclui alguns alimentos fontes de sódio consumidos na região, como a charque.

A pesquisa de orçamentos familiares (POF) aponta que o consumo diário de sódio no período de 20082009 foi de aproximadamente $4700 \mathrm{mg} / \mathrm{dia}$, o equivalente a cerca de $12 \mathrm{~g}$ de sal/ dia, ou seja, mais que o dobro da recomendação diária, sendo a maior parte desse consumo proveniente do sal de adição (IBGE, 2010). Oliveira DC, et al. (2016) ressaltam que a educação em saúde é uma ferramenta importante na prevenção das DCV, pois auxilia na melhora da qualidade de vida e dos hábitos diários, aumentando a prática de atividade física e melhorando o consumo alimentar, especialmente o consumo de sódio através da redução do sal de adição e de alimentos fontes de sódio. Devido a esses aspectos o consumo alimentar de sódio parece estar subestimado nesse estudo, especialmente no grupo B.

No que diz respeito aos alimentos fontes de sódio mais consumidos em ambos os grupos, destaca-se a utilização dos temperos prontos que pode ser favorecida por costumes e hábitos alimentares regionais, bem como pela diminuição da sensibilidade das papilas gustativas, sendo este último um processo considerado normal durante o envelhecimento. Nesse contexto ressalta-se a necessidade da adição de temperos naturais com o objetivo de realçar o sabor das refeições, bem como a potencialidade das atividades de educação em saúde na mudança dos hábitos alimentares na população estudada (GOMES DRP, et al., 2020).

Em relação aos alimentos listados pelo QFA ELSA - versão reduzida observou-se que, mesmo este sendo validado para todo o território nacional, alguns alimentos não compõe os hábitos alimentares do território estudado como, por exemplo, o chimarrão e o acarajé. Além disso, outros alimentos consumidos com frequência na região como a charque e a tapioca não são incluídos no QFA. Portanto sugere-se a necessidade de elaboração e/ou adaptação de um QFA que contemple os hábitos alimentares da região.

No consumo alimentar não houve diferença na ingestão de pães, cereais e tubérculos; carnes, ovos, leite e derivados; frutas; doces e bebidas entre os grupos. Contudo, o grupo A consumiu com maior frequência alimentos dos seguintes grupos: verduras, legumes, leguminosas e massas e outras preparações (Tabela 4). 
Tabela 4 - Frequência alimentar entre os grupos A e B de idosos participantes das atividades grupais do Hiperdia de um município da zona da mata de Pernambuco, 2018.

\begin{tabular}{|c|c|c|c|c|c|c|}
\hline \multirow[t]{2}{*}{ Grupo alimentar } & \multirow[t]{2}{*}{ Alimento fonte } & \multicolumn{2}{|c|}{$\begin{array}{c}\text { Grupo A } \\
n=25\end{array}$} & \multicolumn{2}{|c|}{$\underset{n=25}{\operatorname{Grupo} B}$} & \multirow[t]{2}{*}{ Valor $p$ * } \\
\hline & & Média & DP & Média & DP & \\
\hline \multirow{4}{*}{ Pães, Cereais e Tubérculos } & Arroz & 4,36 & $\pm 1,18$ & 4,24 & $\pm 1,44$ & 0,67 \\
\hline & Tubérculo & 4,56 & $\pm 1,00$ & 4,00 & $\pm 1,, 50$ & 0,16 \\
\hline & Cuscuz & 3,12 & $\pm 1,85$ & 3,40 & $\pm 1,25$ & 0,79 \\
\hline & $\begin{array}{c}\text { Consumo do grupo } \\
\text { alimentar }\end{array}$ & 26,32 & $\pm 8,80$ & 27,24 & $\pm 6,59$ & 0,56 \\
\hline \multirow{4}{*}{ Frutas } & Banana & 2,88 & $\pm 1,96$ & 2,52 & $\pm 1,73$ & 0,51 \\
\hline & Laranja & 2,80 & $\pm 2,06$ & 2,36 & $\pm 1,72$ & 0,42 \\
\hline & Maça/Pera & 2,76 & $\pm 1,80$ & 1,76 & $\pm 1,83$ & 0,06 \\
\hline & $\begin{array}{c}\text { Consumo do grupo } \\
\text { alimentar }\end{array}$ & 15,84 & $\pm 9,46$ & 12,92 & $\pm 6,97$ & 0,28 \\
\hline \multirow{4}{*}{$\begin{array}{l}\text { Verduras, legumes e } \\
\text { leguminosas }\end{array}$} & Feijão & 4,08 & $\pm 1,38$ & 3,56 & $\pm 1,60$ & 0,19 \\
\hline & Tomate & 4,04 & $\pm 1,71$ & 3,20 & $\pm 1,95$ & 0,08 \\
\hline & Cenoura & 3,24 & $\pm 1,92$ & 2,40 & $\pm 2,10$ & 0,19 \\
\hline & $\begin{array}{c}\text { Consumo do grupo } \\
\text { alimentar }\end{array}$ & 37,72 & $\pm 12,6$ & 30,16 & $\pm 10,13$ & 0,02 \\
\hline \multirow{5}{*}{$\begin{array}{l}\text { Ovos/ Carnes } \\
\text { Leite e derivados }\end{array}$} & Frango cozido & 4,04 & $\pm 1,09$ & 3,00 & $\pm 1,19$ & 0,003 \\
\hline & Leite & 2,88 & $\pm 2,22$ & 3,92 & $\pm 1,89$ & 0,11 \\
\hline & Carne Bovina & 2,04 & $\pm 1,54$ & 3,12 & $\pm 1,56$ & 0,02 \\
\hline & Margarina & 1,60 & $\pm 1,95$ & 2,40 & $\pm 2,17$ & 0,16 \\
\hline & $\begin{array}{c}\text { Consumo do grupo } \\
\text { alimentar }\end{array}$ & 13,32 & $\pm 5,61$ & 16,36 & $\pm 4,96$ & 0,06 \\
\hline \multirow{3}{*}{ Massas e outras preparações } & Macarrão & 1,36 & $\pm 1,35$ & 1,96 & $\pm 1,90$ & 0,33 \\
\hline & Sopa & 1,20 & $\pm 1,04$ & 1,40 & $\pm 1,32$ & 0,70 \\
\hline & $\begin{array}{l}\text { Consumo do grupo } \\
\text { alimentar }\end{array}$ & 15,92 & $\pm 4,68$ & 12,00 & $\pm 4,44$ & 0,007 \\
\hline \multirow{3}{*}{ Doces } & Sorvete & 0,12 & $\pm 0,43$ & 0,20 & $\pm 0,64$ & 0,64 \\
\hline & Chocolate & 0,08 & $\pm 0,27$ & 0,08 & $\pm 0,27$ & 1,00 \\
\hline & $\begin{array}{l}\text { Consumo do grupo } \\
\text { alimentar }\end{array}$ & 0,20 & $\pm 0,50$ & 0,28 & $\pm 0,67$ & 0,71 \\
\hline \multirow{4}{*}{ Bebidas } & Suco da fruta & 3,28 & $\pm 2,03$ & 3,12 & $\pm 1,83$ & 0,55 \\
\hline & Café & 4,96 & $\pm 2,01$ & 5,04 & $\pm 2,24$ & 0,43 \\
\hline & Suco Artificial & 0,40 & $\pm 1,00$ & 1,16 & $\pm 1,95$ & 0,31 \\
\hline & $\begin{array}{c}\text { Consumo do grupo } \\
\text { alimentar }\end{array}$ & 9,52 & $\pm 4,12$ & 10,84 & $\pm 4,55$ & 0,34 \\
\hline
\end{tabular}

Legenda: *Mann-Whitney/Wilcoxon Two-Sample Test. Fonte: Campos JO, et al., 2021.

Em um estudo realizado por Secafim MV, et al. (2016) ao avaliar o consumo de frutas em idosos do município de São Caetano do Sul - São Paulo, observou-se que 58\% dos idosos participantes possuíam o consumo de frutas adequado ( 3 ou mais porções diárias). Entretanto, o pesquisador ressalta que o município em questão possui um elevado Índice de Desenvolvimento Humano (IDH: 0,862 em 2010), o que impacta positivamente no consumo alimentar da população, o que difere da população do presente estudo (IDH: 0,640 em 2010) (IBGE, 2010).

Ao comparar os dois grupos estudados, observamos que o grupo A consome com maior frequência verduras, legumes e leguminosas, entre os quais se destacam como alimentos fontes o feijão, o tomate e a cenoura. De acordo com Bina ACO e Abreu WC (2016) a realização das atividades de educação alimentar e nutricional com idosos, afeta diretamente a frequência do consumo de verduras e legumes, favorecendo o aumento no consumo desses alimentos. Esse resultado pode ser observado no grupo A, o qual possui uma maior frequência nas atividades educativas do Hiperdia e consequentemente, maior consumo desses alimentos. 
Ao avaliar o consumo de ovos, carnes, leites e derivados, observou-se que o grupo A consome mais frango e menos carne bovina quando comparado ao grupo B. Mauricio LS, et al. (2014) ao avaliar o consumo de carnes em idosos participantes de um projeto com foco na educação em saúde, observou que os participantes consumiam frequentemente peixes, ovos e frango, além de apresentar um baixo consumo de carne vermelha. Esse resultado corrobora com este estudo sugerindo que as orientações nutricionais realizadas durante 0 Hiperdia impactam positivamente na melhora do consumo alimentar dos idosos.

O grupo B apresentou uma tendência ao maior consumo de margarina/manteiga quando comparado ao grupo A. O Ministério da Saúde recomenda, para os idosos, o consumo de, no máximo, uma porção por dia de óleos e gorduras, além de incentivar o consumo de alimentos fontes de gorduras insaturadas, como o azeite de oliva, e alertar para o consumo de gorduras trans presentes em alimentos como a manteiga e a margarina (PREVIDELI AN, et al., 2017). A orientação sobre o consumo de gorduras é um tema trabalhado nas atividades do grupo Hiperdia, uma vez que essa orientação auxilia no tratamento das DCNT.

No consumo de massas e outras preparações, destacaram-se como alimentos mais consumidos o macarrão e a sopa. No que diz respeito à soma total da frequência de consumo de todos os alimentos desse grupo alimentar, que engloba preparações como peixes fritos e cozidos, frango cozido, peito de frango e sopas, o grupo A apresentou um consumo maior quando comparado ao grupo B.

Assim, o presente estudo sugere que embora não tenha sido observadas diferenças no estado nutricional entre os grupos avaliados, encontraram-se distinções no consumo alimentar, que indicam o maior consumo de alimentos considerados saudáveis no grupo A. Segundo Lima RF, et al. (2016), as intervenções nutricionais não impactam, em curto prazo, no estado nutricional de idosos, entretanto proporcionam melhoras nos hábitos alimentares e no estilo de vida que se torna mais saudável. Este cenário reforça a necessidade de fortalecer o desenvolvimento de atividades de educação em saúde no âmbito da APS, uma vez que é previsto o desenvolvimento de ações de prevenção de agravos e promoção à saúde, relativas aos cuidados nutricionais para grupos de pessoas com doenças específicas, como as DCNT, nesse nível de atenção.

O presente estudo apresenta informações que fornecem embasamento teórico para a elaboração de estratégias e para o fortalecimento das ações educativas desenvolvidas pelo programa Hiperdia. Contribuindo, assim para o cuidado integral da saúde de idosos hipertensos e/ou diabéticos. Ressaltamos que o estudo apresenta como limitação a natureza transversal da pesquisa, não possibilitando uma avaliação do impacto das atividades educativas ao longo do tempo. Além disso, o tamanho amostral, apesar de incluir o total de idosos que participam das atividades educativas do Hiperdia das equipes avaliadas, não permite uma ampliação dos resultados encontrados para toda a população de idosos hipertensos e/ou diabéticos brasileiros.

\section{CONCLUSÃO}

Conclui-se que o grupo A apresentou um maior consumo de verduras, legumes e frango bem como um menor consumo de carne bovina e gorduras, quando comparado ao grupo B. Sugerindo que as atividades educativas quando realizadas de forma regular podem favorecer a melhora no consumo alimentar de idosos e auxiliar no controle dessas patologias. Assim, faz se necessário fortalecer as atividades educativas no programa Hiperdia a fim de obter melhores resultados no tratamento da HAS e do DM. Ressalta-se que as atividades devem abordar as reais necessidades da população alvo e contribuir para o tratamento e para a melhora da qualidade de vida dos participantes.

\section{REFERÊNCIAS}

1. ANDRADE RKO, et al. Estado nutricional de idosos do grupo feliz idade da cidade de capitão Enéas/MG. Revista Multitexto, 2015; 3(1): 73-81.

2. ARAÚJO LSA, et al. Idosos e grupos de convivência: motivos para a não adesão. SANARE, $2017 ; 16$ (1): 58-67.

3. BINA ACO, ABREU WC. A influência da educação nutricional no perfil antropométrico e dietético de idosos fisicamente ativos. Revista Brasileira de Nutrição esportiva, 2016; 10(58): 429-438. 
4. BOTTON A, et al. Diferenças de gênero no acesso aos serviços de saúde: problematizações necessárias. Mudanças: Psicologia da Saúde, 2017; 25(1): 67-72.

5. BRASIL. Ministério da Saúde. Orientações para a coleta e análise de dados antropométricos em serviços de saúde: Norma Técnica do SISVAN. $2011 . \quad$ Disponível em: http://189.28.128.100/dab/docs/portaldab/publicacoes/orientacoes_coleta_analise_dados_antropometricos.pdf. Acessado em: 25 de março de 2021.

6. BRASIL. Ministério da Saúde. Plano de ações estratégicas para o enfrentamento das doenças crônicas não transmissíveis (DCNT) no Brasil 2011-2022. 2011.2 Disponível em: http://bvsms.saude.gov.br/bvs/publicacoes/plano_acoes_enfrent_dcnt_2011.pdf. Acessado em 28 de março de 2021.

7. BRASIL. Ministério da Saúde. Plano de Reorganização da Atenção à Hipertensão arterial e ao Diabetes mellitus: Manual de Hipertensão arterial e Diabetes mellitus. Brasília, 2001. Disponível em: http://bvsms.saude.gov.br/bvs/publicacoes/reorganizacao_plano.pdf. Acessado em: 25 de março de 2021.

8. BRASIL. Ministério da Saúde. Relatório do III fórum de monitoramento do plano de ações estratégicas para o enfrentamento das doenças crônicas não transmissíveis no Brasil. 2018. Disponível em: http://bvsms.saude.gov.br/bvs/publicacoes/relatorio_iii_forum_monitoramento_plano.pdf. Acessado em: 25 de março de 2021.

9. CARDOZO NR, et al. Estado nutricional de idosos atendidos por unidades de saúde da família na cidade de PelotasRS. Sociedade Brasileira de Nutrição Parenteral e enteral, 2017; 32(1): 94-8.

10. CARVALHO ANDA. Importância da redução dos fatores de risco para complicações da hipertensão e do diabetes na qualidade de vida de usuário da Unidade de Saúde Candidés, em Divinópolis Minas Gerais. Trabalho de conclusão especialização (Gestão do cuidado em saúde da família) - Universidade Federal de Minas Gerais - Belo Horizonte, Minas Gerais. 2018; 31 p.

11. CHUMLEA WC, et al. Estimating stature from knee height for persons 60 to 90 years of age. Journal of the American Geriatrics Society, 1985; 33(2): 116-120.

12. DA SILVA IS, et al. Grupo de autocuidado apoiado para portadores de doenças crônicas na atenção primária à saúde: um relato de experiência. Brazilian Journal of Development, 2020; 6(7): 51920-51930.

13. FALSARELLA GR, et al. Envelhecimento e os fenótipos da composição corporal. Revista Kairós: Gerontologia, 2014; 17 (2): 57-77.

14. FECHINE BRA, TROMPIERI N. O processo de envelhecimento: as principais alterações que acontecem com o idoso com o passar dos anos. Revista Científica Internacional, 2012; 20(1): 106-32.

15. GOMES DRP, et al. Avaliação do paladar de idosos e sua relação com estado nutricional e hábitos alimentares. Pan American Journal of Aging Research, 2020; 8(1): e37707.

16. IBGE. Pesquisa de Orçamentos Familiares 2008-2009 - POF. Análise do consumo alimentar pessoal no Brasil. Rio de Janeiro, 2010: 1-150.

17. Instituto Brasileiro de Geografia e Estatística (IBGE). Índice de desenvolvimento humano- cidades: Vitória de Santo Antão-PE e São Caetano do Sul-SP. 2010. Disponível em: https://cidades.ibge.gov.br/. Acessado em 27 de março de 2021

18. LIMA RF, et al. Impacto da intervenção nutricional sobre o prognóstico clínico de pacientes com o risco cardiovascular elevado. Anais VIII SIMPAC, 2016; 8(1): 494-500.

19. LIPSCHITZ D. Screening for nutritional status in the elderly. Primary Care,1994; 21(1): 55-67.

20. MANNATO LW. Questionário de frequência alimentar ELSA-Brasil: proposta de redução e validação da versão reduzida. Dissertação (Mestrado em Saúde Coletiva) - Centro de Ciências da Saúde, Universidade Federal do Espírito - Vitória, Espírito Santo. 2013; 118 p.

21. MAURICIO LS, et al. Avaliação da frequência de consumo alimentar de idosos participantes do projeto integrar. Revista Redes de cuidado a saúde, 2014; 8(1): 1-14.

22. OLIVEIRA DC, et al. Educação em saúde na prevenção de doenças cardiovasculares: um estudo de intervenção. Revista Diálogos Acadêmicos, 2016; 5(1): 1-13.

23. OLIVEIRA HHB, et al. Risco cardiovascular e composição corporal de idosas participantes de projeto de atividades físicas e recreativas. Brazilian Journal of Development, 2020; 6 (3): 16437-16448.

24. Organização Mundial da Saúde (OMS). Resumo relatório mundial de envelhecimento e saúde. 2015. Disponível em: https://sbgg.org.br/wp-content/uploads/2015/10/OMS-ENVELHECIMENTO-2015-port.pdf. Acessado em: 29 de abril de 2021.

25. OLIVEIRA VB, et al. Risco cardiovascular, indicadores antropométricos e mini avaliação nutricional reduzida: associação com índice de massa corporal na avaliação nutricional de idosos. Nutr. clín. diet. Hosp., 2019; 39(1): 6975.

26. PIOVESANA PM. Consumo de sal e sensibilidade gustativa entre pacientes hipertensos no contexto da síndrome metabólica. Tese (Doutorado em Enfermagem) - Faculdade de Enfermagem, Universidade estadual de Campinas Campinas, São Paulo. 2015; 128 p.

27. PODMELLE RM, ZIMMERMANN RD. Fatores influentes no estilo de vida e na saúde dos idosos brasileiros: uma revisão integrativa. Estudos Interdisciplinares sobre o Envelhecimento, 2018; 23(1): 103-123.

28. PREVIDELLI NA, et al. Balanço de macronutrientes na dieta de idosos brasileiros: análises da Pesquisa Nacional de Alimentação 2008-2009. Revista Brasileira de Epidemiologia, 2017; 20(1):70-80.

29. ROCHA TAH, SILVA NC. Oferta de cuidado primário para doenças crônicas: uma análise da eficiência técnicas das equipes de saúde brasileiras. APS em Revista, 2019; 1(1): 18-28.

30. SBC. Diretrizes Brasileiras de Hipertensão-2020. Arquivos brasileiros de Cardiologia, 2020; 116(3): 516-658. 
31. SECAFIM MV, et al. Avaliação do consumo de frutas por idosos de São Caetano do Sul, São Paulo. Geriatria e gerontologia aging, 2016; 10(2): 57-63.

32. SILVA GSM, et al. Efeitos de um programa de intervenção de atividade física, educação e promoção de saúde com idosos hipertensos usuários do Sistema Único de Saúde. Revista Eletrônica Acervo Saúde, 2021; 13 (4): e6926.

33. SILVA TA, et al. Relação entre o envelhecimento, a polimedicação e o enfermeiro: Uma Revisão Bibliográfica. Revista de Iniciação Científica e Extensão, 2020; 3 (2): 438-56.

34. SILVA, JVF, et al. A relação entre o envelhecimento populacional e as doenças crônicas não transmissíveis: sério desafio de saúde pública. Ciências biológicas e da saúde. Maceió, 2015, 2 (3): 91-100.

35. SILVEIRA EA, et al. Acurácia de pontos de corte de IMC e circunferência da cintura para a predição de obesidade em idosos. Ciência \& Saúde Coletiva, 2020; 25 (1):1073-82.

36. SIMIELI I, et al. Realidade do envelhecimento populacional frente às doenças crônicas não transmissíveis. Revista Eletrônica Acervo Saúde, 2019; 37: e1511.

37. SOUZA AM, et al. Marcadores de risco cardiovascular em idosos da Estratégia de Saúde da Família. Brazilian Journal of Development, 2020; 6(6): 36094-109.

38. SOUZA IP, et al. Avaliação do perfil antropométrico e a presença de sarcopenia em um grupo de idosas da cidade de Fortaleza-CE. Revista saúde e ciência. 2018; 7(1): 15-25.

39. VITOLO MR. Nutrição: da gestação ao envelhecimento. 2 nd ed. Rio de Janeiro. 2015; 554p. 